\title{
3D EIT image reconstruction with GREIT
}

\author{
Bartłomiej Grychtol ${ }^{1}$, Beat Müller ${ }^{2}$, Andy Adler $^{3}$ \\ ${ }^{1}$ Fraunhofer Project Group for Automation in Medicine and Biotechnology, Mannheim, Germany b.grychtol@ipa.fraunhofer . de \\ ${ }^{2}$ Swisstom AG, Landquart, Switzerland \\ ${ }^{3}$ Systems and Computer Engineering, Carleton University, Ottawa, Canada
}

\begin{abstract}
The Graz consensus reconstruction algorithm for EIT (GREIT) has become popular in lung EIT. One shortcoming of the original formulation of GREIT is its restriction to $2 \mathrm{D}$ image reconstruction and so planar electrode arrangement. We present an extension of the GREIT algorithm to 3D and discuss some of the issues that arise.
\end{abstract}

\section{Introduction}

Since its publication in 2009, the Graz consensus reconstruction algorithm for EIT (GREIT) [1] has become popular in lung EIT. The original formulation and implementation available in EIDORS (www.eidors.org) was limited to cylindrical geometries with planar electrode arrangement. Subsequent adaptations allowed reconstructions on arbitrary geometry [2]. Recently, Ahn et al. [3] proposed and tested in a 360-electrode micro-EIT setup an extension of the GREIT algorithm to 3D. We describe a similar implementation contributed to EIDORS and investigate the algorithm's properties. We use a commercially available 32-electrode EIT system intended for lung imaging.

\section{Methods}

GREIT is a linear reconstruction for difference EIT. Images are obtained by multiplying difference voltage data by a reconstruction matrix $\mathbf{R}$ calculated from $k$ (simulated) measurements $\mathbf{y}$ of point target contrasts and the corresponding 'desired images' $\tilde{\mathbf{x}}$ as

$$
\mathbf{R}=\underset{\mathbf{R}}{\arg \min } \sum_{k}\left\|\tilde{\mathbf{x}}^{(k)}-\mathbf{R} \mathbf{y}^{(k)}\right\|_{\mathbf{W}^{(k)}}^{2}
$$

where $\mathbf{w}^{(k)}$ is a vector weighting individual desired image elements and is commonly set to a scalar value identical for all desired images [1, eq. 13].

One key parameter influencing the properties of the GREIT reconstruction matrix is the definition of the desired images. In the original formulation, they are circles centered at the point targets' position, with an inner circular zone where the amplitude is flat and a zone outside a larger circular boundary where the amplitude is zero. Between the two circles, the amplitude is supposed to "gradually decrease" [1]. We propose to formalize the desired image definition by means of a sigmoid function of position

$$
f(\mathbf{r})=\left(1+e^{s\left(\left|\mathbf{r}-\mathbf{r}_{0}\right|-R\right)}\right)^{-1},
$$

where $\mathbf{r}$ is the position vector in $2-$ or $3 \mathrm{D}, \mathbf{r}_{0}$ is the target center, $R$ is the desired radius and $s$ is a scalar controlling the blur (fig. 1). $f(\mathbf{r})$ can be discretized on pixels or voxels.

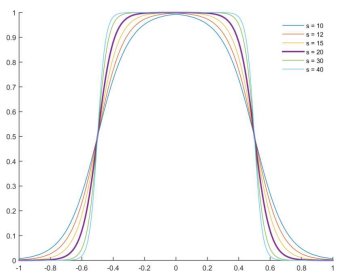

(a) $R=0.5$



(b) $R=0.25$
Figure 1: Plots of the function $f(x)=1 /\left(1+e^{s(|x|-R)}\right)$ for different values of $s$ with the default value indicated in bold.

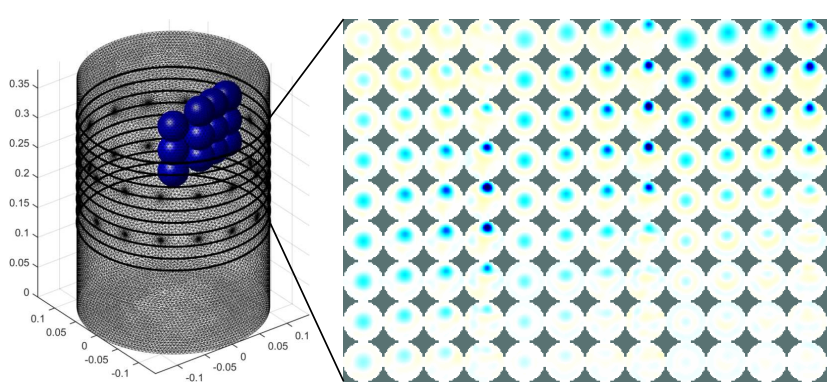

Figure 2: Left: FE model of a water tank showing cut planes between voxel layers and positions of a non-conductive spherical target. Right: images reconstructed using the proposed algorithm. Each row corresponds to one voxel layer, and each column to a different target position.

\section{Discussion}

Sample 3D reconstruction of data collected with the Pioneer Set (Swisstom AG) from a water tank with two layers of 16 electrodes are shown in fig. 2. Variations in vertical and radial position of a non-conductive spherical target are successfully reconstructed. As expected, amplitude response and resolution fall toward the long axis of the tank and away from the electrode plane. On the other hand, ringing seems to be stronger in the electrode planes.

Extension of the GREIT framework to 3D expands the application of this popular lung EIT algorithm to other domains, such as head or breast imaging. It also rises interesting questions: What is the desired image for an out-of-plane target? How, if at all, should the desired image amplitude be affected by the measurement sensitivity? Is noise figure a suitable way to choose the hyperparameter? How many targets are necessary, and how should they be distributed? Are the answers dependent on the stimulation pattern? As experience with 3D imaging in the EIT community grows, we antipate that solutions will be developed for these issues.

\section{References}

[1] Adler A, Arnold JH, Bayford R, et al. Physiological measurement 30(6):S35-55, 2009

[2] Grychtol B, Lionheart WRB, Wolf GK, et al. In 12th International Conference on Electrical Impedance Tomography. Bath, UK, 2011

[3] Ahn S, Wi H, Oh TI, et al. Journal of Applied Mathematics 2014(Article ID 562176):1-11, 2014 